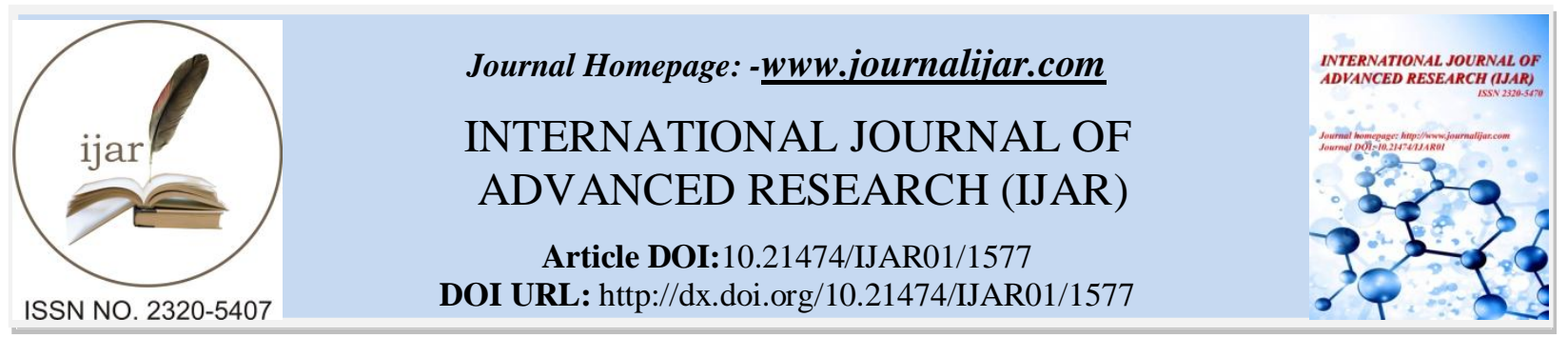

RESEARCH ARTICLE

\title{
EFFECTIVENESS OF PLAY THERAPY ON PHYSIOLOGICAL PARAMETERS OF HOSPITALIZED CHILDREN AND ANXIETY LEVEL OF THEIR PARENTS.
}

1. Lecturer, C.O.N. CIMS

2. Associate Professor HCN, SRHU.

3. Sr. Tutor, HCN, SRHU.

\section{Manuscript Info}

Manuscript History

Received: 16 July 2016

Final Accepted: 22 August 2016

Published: September 2016

Key words:-

Anxiety, Hospitalized children, Play therapy, Parents, and Physiological parameters.

\section{Abstract}

An experimental study was conducted in a pediatric ward at Himalayan Hospital jolly grant. Dehradun Uttarakhand to assess the effectiveness of play therapy on physiological parameters of hospitalized children and anxiety level of their parents. Research design was two group pre test-posttest designs. Sample size was 60 hospitalized children between the age group of 3-8 years and their parents (30 in experimental group and 30 in control group) who fulfilled inclusion criteria were selected through simple random sampling technique and they were randomly divided in to experimental and control group. Ethical permission obtained and the data was collected by using Beck Anxiety Inventory scale to assess the level of anxiety among parents and physiological parameters of children. Intervention was given in the form of play therapy to experimental group for one week. The mean post test anxiety score after play therapy in the control group was $29.63 \pm 12.5$ and in experimental group , it was $20.87 \pm 8.8$. The result shows a significance difference between the mean anxiety score in control and experimental group $(\mathrm{p}<0.05)$. Play therapy was also effective in improving the physiological parameters of hospitalized children. The finding of the study reveals that the play therapy had a vital role in improving the physiological parameters of hospitalized children and in reducing the level of anxiety of parents.

Copy Right, IJAR, 2016,. All rights reserved.

\section{Introduction:-}

Hospitalization is considered as a stressful event for children; the environment which surrounds the children in a hospital, physical conditions such as pain and underlying disease, hospital procedures such as blood tests, cannulization or even a medical examination in the hospital could be a stressor for children. ${ }^{\mathrm{i}}$. Research supports the effectiveness of play therapy with children experiencing a wide variety of social, emotional, behavioral, and learning problems, including: children whose problems are related to life stressors, such as divorce, death, relocation, hospitalization, chronic illness, assimilate stressful experiences, physical and sexual abuse, domestic violence, and natural disasters. ${ }^{\text {ii }}$ 
Play therapy may also be used to reduce stress, promote cognitive development and provide insight about and resolution of inner conflicts or dysfunctional thinking in the child. ${ }^{\mathrm{iii}}$

\section{Need of the study:-}

Play therapy is generally employed with children aged 3 to 11 and provides a way for them to express their experiences and feelings through a natural, self-guided, self-healing process. ${ }^{\text {iv }}$ About 30 percent of children are hospitalized at least once during their childhood in the hospital; about 5 percent of them are hospitalized several times. ${ }^{v}$ Research supports the effectiveness of play therapy with children experiencing a wide variety of social, emotional, behavioral, and learning problems, including: children whose problems are related to life stressors, such as divorce, death, relocation, hospitalization, chronic illness, assimilate stressful experiences, physical and sexual abuse, domestic violence, and natural disasters. ${ }^{\text {vi }}$

\section{Problem statement:-}

A study to assess the effectiveness of play therapy on physiological parameters of hospitalized children and anxiety level of their parents in a selected hospital of Dehradun, Uttarakhan

\section{Objective:-}

1. To evaluate the effectiveness of play therapy on physiological parameters of children and on level of anxiety of their parents.

2. To find association between socio-demographic variables with level of anxiety of parents.

\section{Hypotheses:-}

1. $1 \mathrm{H}_{1}$ : There would be significant difference in physiological parameters of children after play therapy in experimental group than the control group.

2. $2 \mathrm{H}_{1}$ : The mean post test score of level of anxiety of parents will be lower than the pretest score.

3. $3 \mathrm{H}_{1}$ : There would be significant association between the level of anxiety of parents and selected sociodemographic variables

\section{Conceptual Framework}

The conceptual frame work selected for this study was based on the system models derived from the Ludwig Von Bertalanffy's general system theory. In this model a system is made up of separate components. The theory explains the relationship between wholes and parts describes concepts and predicts how the parts will behave and react.

\section{Material and mehods:-}

The research design used in this study was quasi-experimental in nature. The study was conducted at pediatric ward at jollygrant Dehradun, Uttarakhand. An experimental approach with two group pre test-posttest design was used as research design for the study. The sample size was 60 hospitalized children between the age group of 3-8 years and their parents (30 in experimental group and 30 in control group) who fulfilled inclusion criteria, were selected through simple random sampling technique and they were randomly divided in to experimental and control group .The data was collected by using Beck Anxiety Inventory scale to assess the level of anxiety of parents and physiological parameters of children. Intervention was given in the form of play therapy to experimental group for one week. The obtained data was analyzed in terms of objectives and hypothesis by using descriptive and inferential statistics.

\section{Result and findings:-}

Related to Socio demographic variables of samples:-

Data presented in table no.1 shows that most of children 17 (57\%) were belongs between the age group of 6-8 years, in experimental group where as $17(57 \%)$ were between 3-5 years in control group. Most of the children 21 (70\%) were male in experimental group and 17(56.7\%) male in control group. Most of the Information 21(70\%) was given by the mothers in experimental group and in control group both the informers were mother and father constitute equal percentage that is 15(50\%).Regarding number of children in family, in experimental group 11(37\%) families had 3 children where as in control group 15 (50\%) families had 2 children. Most of parents 20 (70\%) were between the age group 31-40 years in experimental group where as 15(50\%) were between 20-30 years in control group. Most of fathers, nine (30\%) had graduate education in experimental group where as in control group 10 
(33.3\%) had secondary education. About the mothers $12(46 \%)$ were graduate in experimental group where as in control group $10(33 \%)$ were graduate.

Table no.1:-Frequency, percentage and distribution of socio-demographic variables

$\mathrm{N}=\mathbf{6 0}$

\begin{tabular}{|c|c|c|c|c|c|c|c|}
\hline \multirow[t]{2}{*}{ CHARACTERSTICS } & \multicolumn{2}{|c|}{ EXP GROUP } & \multicolumn{2}{|c|}{ CONTROL GROUP } & \multicolumn{2}{|c|}{ TOTAL } & \multirow[t]{2}{*}{ P value } \\
\hline & $\mathbf{f}$ & $\%$ & $\mathbf{f}$ & $\%$ & $\mathbf{f}$ & $\%$ & \\
\hline $\begin{aligned} \text { Age of child } \\
$\[ \quad 3-5 \text { years } \]$ \\
\bullet \quad 6-8 \text { years }\end{aligned}$ & $\begin{array}{l}13 \\
17\end{array}$ & $\begin{array}{l}43.3 \\
\mathbf{5 6 . 7}\end{array}$ & $\begin{array}{l}17 \\
13\end{array}$ & $\begin{array}{l}\mathbf{5 6 . 7} \\
43.3\end{array}$ & $\begin{array}{l}30 \\
30\end{array}$ & $\begin{array}{l}50 \\
50\end{array}$ & 0.30 \\
\hline $\begin{array}{cl}\text { Gender of child } \\
\bullet & \text { Male } \\
\bullet & \text { Female }\end{array}$ & $\begin{array}{c}21 \\
9\end{array}$ & $\begin{array}{l}70 \\
30\end{array}$ & $\begin{array}{l}17 \\
13\end{array}$ & $\begin{array}{l}\mathbf{5 6 . 7} \\
43.3\end{array}$ & $\begin{array}{l}38 \\
22\end{array}$ & $\begin{array}{l}63 \\
37\end{array}$ & 0.28 \\
\hline $\begin{array}{cl}\text { Information received from } \\
\qquad \quad \text { Mother } \\
\bullet \quad \text { Father }\end{array}$ & $\begin{array}{l}21 \\
09 \\
\end{array}$ & $\begin{array}{l}70 \\
30 \\
\end{array}$ & $\begin{array}{l}15 \\
15 \\
\end{array}$ & $\begin{array}{l}50 \\
50 \\
\end{array}$ & $\begin{array}{l}36 \\
24 \\
\end{array}$ & $\begin{array}{c}60 \\
40 \\
\end{array}$ & 0.11 \\
\hline $\begin{array}{cl}\text { No of children in family } \\
\text { - } & \text { One } \\
\text { - } & \text { Two } \\
\text { - } & \text { Three } \\
\text { - } & \text { four and above } \\
\end{array}$ & $\begin{array}{l}03 \\
09 \\
11 \\
07\end{array}$ & $\begin{array}{l}10 \\
30 \\
\mathbf{3 7} \\
23\end{array}$ & $\begin{array}{l}04 \\
15 \\
06 \\
05\end{array}$ & $\begin{array}{c}13.3 \\
\mathbf{5 0} \\
20 \\
16.7\end{array}$ & $\begin{array}{l}07 \\
24 \\
17 \\
12\end{array}$ & $\begin{array}{l}12 \\
40 \\
28 \\
20\end{array}$ & 0.32 \\
\hline 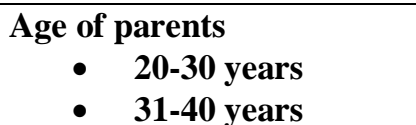 & $\begin{array}{l}10 \\
20\end{array}$ & $\begin{array}{l}30 \\
70\end{array}$ & $\begin{array}{l}15 \\
15\end{array}$ & $\begin{array}{l}\mathbf{5 0} \\
\mathbf{5 0}\end{array}$ & $\begin{array}{l}25 \\
35\end{array}$ & $\begin{array}{l}42 \\
58\end{array}$ & 0.19 \\
\hline $\begin{aligned} & \text { Education status of father } \\
& \text { - } \text { Post graduate and } \\
& \text { above } \\
& \text { - } \text { Graduate } \\
& \text { - } \text { Secondary } \\
& \text { - } \text { Primary } \\
& \text { - } \text { Illiterate }\end{aligned}$ & $\begin{array}{l}04 \\
09 \\
06 \\
08 \\
03\end{array}$ & $\begin{array}{l}13 \\
\\
\mathbf{3 0} \\
20 \\
27 \\
10\end{array}$ & $\begin{array}{l}05 \\
\\
07 \\
10 \\
07 \\
01\end{array}$ & $\begin{array}{c}16.7 \\
23.3 \\
\mathbf{3 3 . 3} \\
23.3 \\
3.3\end{array}$ & $\begin{array}{l}09 \\
16 \\
16 \\
15 \\
04\end{array}$ & $\begin{array}{c}15 \\
26.66 \\
26.66 \\
25 \\
6.66\end{array}$ & 0.65 \\
\hline \begin{aligned} & \multicolumn{2}{l}{ Education status of } \\
& mother \\
& - Post graduate and \\
& above \\
& - Graduate \\
& - Secondary \\
& - Primary \\
& - Illiterate \\
&\end{aligned} & $\begin{array}{l}- \\
12 \\
05 \\
08 \\
05\end{array}$ & $\begin{array}{c}- \\
\mathbf{4 0 . 0} \\
16.7 \\
26.7 \\
16.7\end{array}$ & $\begin{array}{l}01 \\
\\
10 \\
09 \\
07 \\
03\end{array}$ & $\begin{array}{c}3.3 \\
\\
\mathbf{3 3 . 3} \\
30.0 \\
23.4 \\
10.0\end{array}$ & $\begin{array}{l}01 \\
\\
22 \\
14 \\
15 \\
08\end{array}$ & $\begin{array}{l}02 \\
37 \\
23 \\
25 \\
13\end{array}$ & 0.57 \\
\hline
\end{tabular}


Table no.2:-Frequency, percentage and distribution of socio-demographic variables.

\begin{tabular}{|c|c|c|c|c|c|c|c|}
\hline \multirow[t]{2}{*}{ Characteristics } & \multicolumn{2}{|c|}{$\begin{array}{c}\text { Experimental } \\
\text { group }\end{array}$} & \multicolumn{2}{|c|}{ control group } & \multicolumn{2}{|c|}{ total } & \multirow[t]{2}{*}{ p value } \\
\hline & $\mathrm{f}$ & $\%$ & f & $\%$ & $\mathrm{f}$ & $\%$ & \\
\hline $\begin{array}{c}\text { Occupation of father } \\
\qquad \quad \text { Employed } \\
\bullet \quad \text { Unemployed }\end{array}$ & $\begin{array}{l}16 \\
14\end{array}$ & $\begin{array}{l}53 \\
47\end{array}$ & $\begin{array}{l}11 \\
19\end{array}$ & $\begin{array}{l}37 \\
\mathbf{6 3}\end{array}$ & $\begin{array}{l}27 \\
33\end{array}$ & $\begin{array}{l}45 \\
55\end{array}$ & 0.19 \\
\hline $\begin{array}{c}\text { Occupation of mother } \\
-\quad \text { Employed } \\
-\quad \text { Unemployed }\end{array}$ & $\begin{array}{l}05 \\
25\end{array}$ & $\begin{array}{l}17 \\
\mathbf{8 3}\end{array}$ & $\begin{array}{l}07 \\
23\end{array}$ & $\begin{array}{l}23 \\
77\end{array}$ & $\begin{array}{l}12 \\
48\end{array}$ & $\begin{array}{l}20 \\
80\end{array}$ & 0.51 \\
\hline $\begin{aligned} & \text { Type of family } \\
& \bullet \text { Joint } \\
& \bullet \text { Nuclear } \\
& \bullet \text { Extended }\end{aligned}$ & $\begin{array}{c}11 \\
19 \\
-\end{array}$ & $\begin{array}{c}37 \\
63 \\
-\end{array}$ & $\begin{array}{l}03 \\
26 \\
01\end{array}$ & $\begin{array}{l}10 \\
87 \\
03\end{array}$ & $\begin{array}{l}14 \\
45 \\
01\end{array}$ & $\begin{array}{l}23 \\
75 \\
02\end{array}$ & 0.03 \\
\hline $\begin{array}{c}\text { Monthly family income } \\
\begin{array}{cc}\text { - } & 1000-20,000 \\
\text { - } & 21000-40,000 \\
\text { - } & 41000-60,000 \\
\end{array}\end{array}$ & $\begin{array}{l}18 \\
08 \\
04\end{array}$ & $\begin{array}{l}\mathbf{6 0} \\
27 \\
13\end{array}$ & $\begin{array}{l}20 \\
05 \\
05\end{array}$ & $\begin{array}{l}\mathbf{6 6 . 6} \\
16.7 \\
16.7\end{array}$ & $\begin{array}{l}38 \\
13 \\
09\end{array}$ & $\begin{array}{l}63 \\
22 \\
15\end{array}$ & 0.63 \\
\hline $\begin{array}{l}\text { Previous experience of } \\
\text { hospital } \\
\text { - Never } \\
\text { - Once } \\
\text { - Twice } \\
\text { - } \quad \text { Thrice }\end{array}$ & $\begin{array}{l}09 \\
09 \\
08 \\
04\end{array}$ & $\begin{array}{l}30 \\
30 \\
27 \\
13\end{array}$ & $\begin{array}{l}02 \\
15 \\
10 \\
03\end{array}$ & $\begin{array}{l}\mathbf{0 7} \\
\mathbf{5 0} \\
33 \\
10\end{array}$ & $\begin{array}{l}11 \\
24 \\
18 \\
07\end{array}$ & $\begin{array}{l}18 \\
40 \\
30 \\
12\end{array}$ & 0.09 \\
\hline $\begin{aligned} & \text { Social status of child } \\
&$\[ \quad \text { Playing alone } \]$ \\
&-\quad \text { Playing with sibling } \\
& \text { - } \text { Playing while seeing } \\
& \text { other } \\
& \text { - } \text { Playing not at all }\end{aligned}$ & $\begin{array}{l}04 \\
24 \\
01 \\
01\end{array}$ & $\begin{array}{l}14 \\
\mathbf{8 0} \\
03 \\
03\end{array}$ & $\begin{array}{l}- \\
29 \\
- \\
01\end{array}$ & $\begin{array}{l}- \\
97 \\
- \\
03\end{array}$ & $\begin{array}{l}04 \\
53 \\
01 \\
02 \\
0\end{array}$ & $\begin{array}{l}07 \\
88 \\
02 \\
03\end{array}$ & 0.09 \\
\hline
\end{tabular}

Data presented in table no.2 shows that most of the fathers $16(53 \%)$ were employed. in experimental group where as in control group14 (47\%) were unemployed. Regarding mothers majority of them $25(83 \%)$ were employed in experimental group where as in control group most of $23(77 \%)$ were unemployed. Most of the children $19(63 \%)$ in experimental group and majority of children $26(87 \%)$ in control group belongs to nuclear family. Most of the family $18(60 \%)$ had monthly income about 1000-20,000 rupees in experimental group where as in control group 20 (67\%) had monthly income about 1000-20,000 rupees. In experimental group most of the children nine (30\%) had never been hospitalized previously and nine children (30\%) had previous exposure of hospitalization at once where as in control group 15 children $(50 \%)$ had been admitted once. Majority of children $24(80 \%)$ were playing with their siblings in experimental group where as $29(98 \%)$ in control group. 
Efectiveness of play therapy:-

Table no 3:-Mean, standard deviation and comparison of physiological parameters between pre and posttest effectiveness of play therapy among experimental group.

\begin{tabular}{|l|c|c|c|c|}
\hline \multirow{2}{*}{ PARAMETERS } & \multicolumn{2}{|c|}{ EXPERIMENTAL GROUP } & P & t-value \\
calculated & & \\
\cline { 2 - 3 } & $\begin{array}{c}\text { Pre test } \\
\text { Mean } \pm \text { SD }\end{array}$ & $\begin{array}{c}\text { Post test } \\
\text { Mean } \pm \text { SD }\end{array}$ & & \\
\hline Heart rate & $98.73 \pm 18.751$ & $105.73 \pm 7.930$ & 1.993 & 0.56 \\
\hline SBP & $98.37 \pm 22.182$ & $103.73 \pm 9.285$ & 1.321 & 1.321 \\
\hline DBP & $61 \pm 15.136$ & $66.80 \pm 7.402$ & 2.004 &. $\mathbf{0 5 5}$ \\
\hline Respiratory rate & $22.60 \pm 5.757$ & $23.53 \pm 2.556$ & .970 & .340 \\
\hline SPO2 & $90.03 \pm 4.319$ & $96.37 \pm 2.371$ & 9.451 & $\mathbf{. 0 0 1}$ \\
\hline
\end{tabular}

$\mathrm{df}_{29}=\mathbf{2 . 0 4 5}$ significant

Table no 3shows that the mean pretest heart rate of the experimental group was 98.73 and the post test heart rate was 105.73. The mean pretest systolic blood pressure of the experimental group was 98.73 and the posttest score was 103.73. The mean pretest diastolic blood pressure of the experimental group was 61 and the posttest score was 66.80. The mean pretest respiratory rate was 22.60 and the posttest rate was 23.53 . The mean pretest Spo2 was 90.03 and the posttest Spo2 was 96.37 . These indicate play therapy was effective in improving the spo2 and disystolic blood pressure at the significant level of $\mathrm{P}<0.05$.

Table 4:-mean, standard deviation and comparison of physiological parameters between pre and posttest effectiveness of play therapy among control group using paired t-test.

\begin{tabular}{|c|c|c|c|c|}
\hline \multirow{3}{*}{ PARAMETERS } & \multirow{2}{*}{\multicolumn{2}{|c|}{ CONTROL GROUP }} & & \\
\hline & & & t-value & $P$ value \\
\hline & $\begin{array}{c}\text { Pre test } \\
\text { Mean } \pm \text { SD }\end{array}$ & $\begin{array}{c}\text { Post test } \\
\text { Mean } \pm \text { SD }\end{array}$ & calculated & \\
\hline Heart rate & $105.83 \pm 15.184$ & $107.07 \pm 15.051$ & .577 & 0.568 \\
\hline SBP & $99.00 \pm 16.515$ & $96.73 \pm 14.588$ & 1.543 & .134 \\
\hline DBP & $62.07 \pm 11.259$ & $60.47 \pm 7.496$ & 1.099 & .281 \\
\hline Respiratory rate & $26.60 \pm 7.030$ & $25.87 \pm 5.704$ & .924 & .363 \\
\hline SPO2 & $91.30 \pm 4.340$ & $93.30 \pm 3.798$ & 2.899 & .007 \\
\hline
\end{tabular}

df $_{29}=\mathbf{2 . 0 4 5}$ significant

table no 4 shows that in control group there was no improvement in physiological parameters accept the SPO2. The mean pretest $\mathrm{Spo} 2$ was 91.30 and the posttest mean was 93.30.only $\mathrm{Spo}_{2}$ level was significant in control group. The values were significant at $\mathrm{P}<0.05$ levels

Table 5:- Mean Standard deviation and comparison of physiological parameters of hospitalized children among experimental and control group.

\begin{tabular}{|l|c|c|c|c|}
\hline \multirow{2}{*}{ PARAMETERS } & $\begin{array}{c}\text { EXPERIMENTAL } \\
\text { GROUP }\end{array}$ & CONTROL GROUP & $\begin{array}{c}\text { t-value } \\
\text { calculated }\end{array}$ & \multirow{2}{*}{ P value } \\
\cline { 2 - 3 } & Mean \pm SD & Mean \pm SD & & \\
\hline Heart rate & $105.73 \pm 7.930$ & $107.07 \pm 15.051$ & .429 & 0.669 \\
\hline SBP & $103.73 \pm 16.515$ & $96.73 \pm 14.588$ & 2.217 & $\mathbf{. 0 3 1}$ \\
\hline DBP & $66.07 \pm 7.402$ & $60.47 \pm 7.496$ & 3.293 & $\mathbf{. 0 0 2}$ \\
\hline Respiratory rate & $23.53 \pm 2.556$ & $25.87 \pm 5.704$ & 2.045 & $\mathbf{. 0 4 5}$ \\
\hline SPO2 & $96.37 \pm 2.371$ & $93.30 \pm 3.798$ & 3.752 & $\mathbf{. 0 0 1}$ \\
\hline
\end{tabular}

$\mathrm{DF}_{58}=2.00$ significant* $\mathrm{p}<0.05$

The above table 5 .Result shows that there was improvement in physiological parameters except heart rate at the significant level of $\mathrm{p}<0.05$. 
Effectiveness of play therapy on level of anxiety of parents:-

Figure 1:-Frequency and percentage distribution of pre and posttest scores of level of anxiety of parents among experimental group.

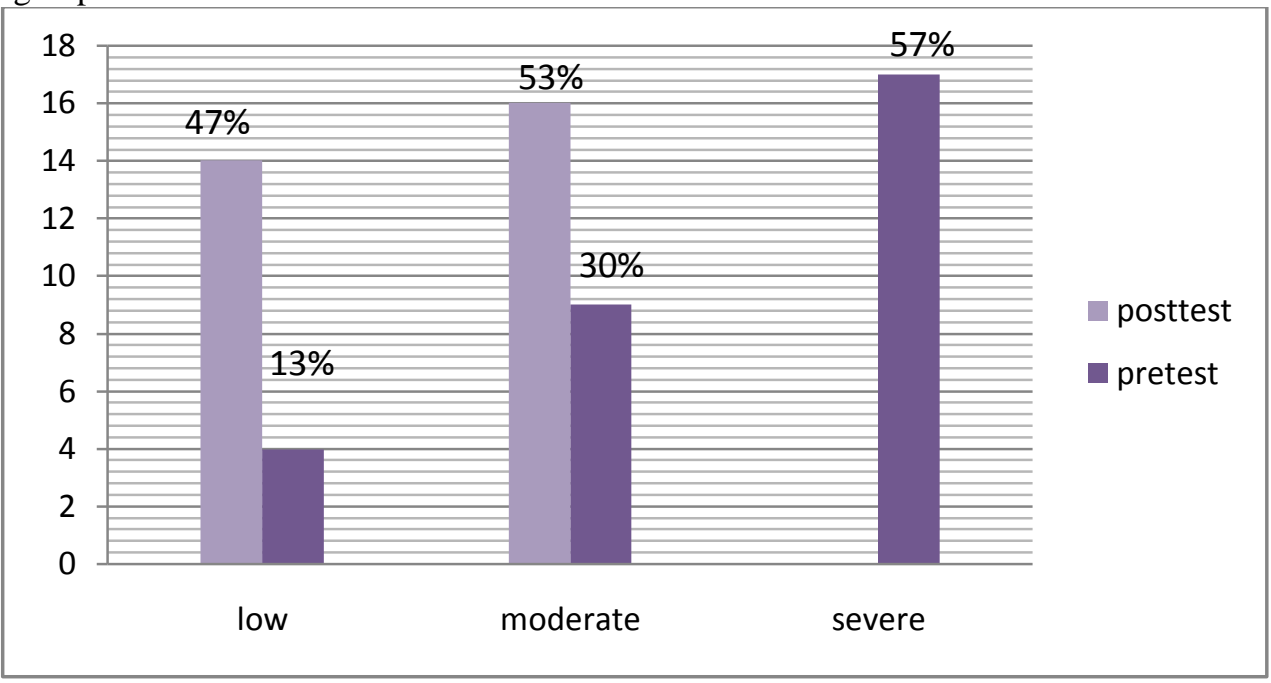

Level of anxiety in experimental group

Graph shows that during in pretest, most of the sample, 17(57\%) had severe anxiety, nine (30\%) had moderate and four (13\%) had low anxiety. In experimental group most of sample, 16(53\%) had moderate anxiety,14 (47\%) had low anxiety. None of them had severe anxiety. This indicates, after intervention there was reduction in the parental anxiety.

Figure 2:- Frequency and percentage distribution of pre and posttest scores of level of anxiety of parents in control group

Graphshows that during pretest, most of the parents, 18(60\%) had severe anxiety, five (17\%) had moderate and seven (20\%) had low anxiety. After 7 days in posttest, most of the parents $11(37 \%)$ had severe anxiety, $11(37 \%)$ had moderate anxiety and $06(26 \%)$ had low anxiety. This indicates that there was also reduction in parental anxiety in control group

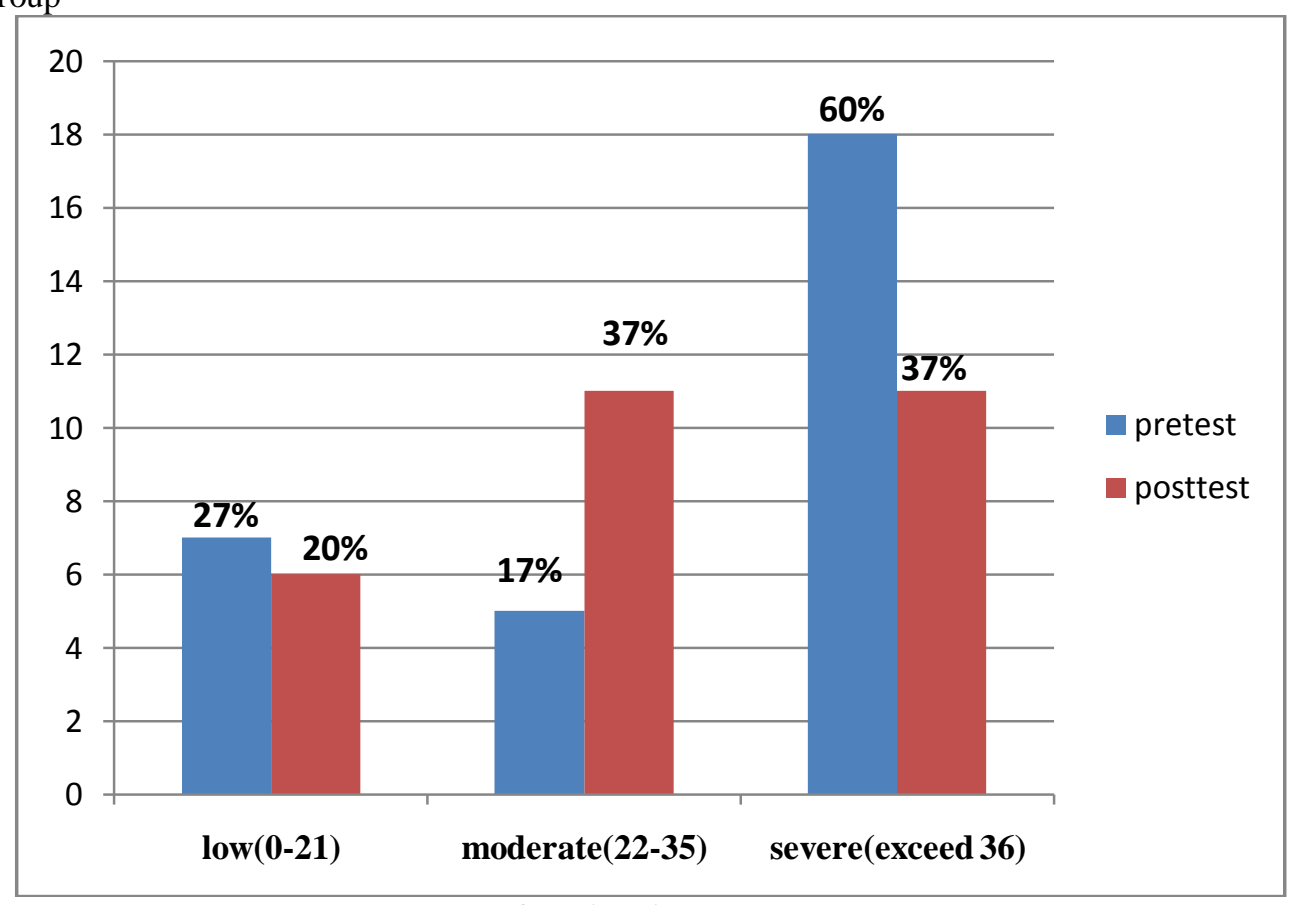

Level of anxiety in control group 
Table6:-mean, standard deviation and comparison between pre and posttest effectiveness of play therapy on anxiety level of parents among experimental group-

\begin{tabular}{|c|c|c|c|c|c|}
\hline \multirow{3}{*}{ S.no } & \multirow{3}{*}{ Group } & & \multicolumn{3}{|c|}{$N=30$} \\
\hline & & Pre anxiety score & Post anxiety score & Paired & P value \\
\hline & & Mean \pm SD & Mean \pm SD & 't'test & \\
\hline 1. & $\begin{array}{l}\text { Experimental } \\
\text { group }\end{array}$ & $34.40 \pm 11.7$ & $20.87 \pm 8.8$ & 8.328 & 0.001* \\
\hline
\end{tabular}

$\mathrm{Df}_{29}=\mathbf{2 . 0 4 5} *$ Significant

Table no 6-, shows that in experimental group mean pre anxiety score was 34.40 and mean post score was 20.87 . The values were significant at $\mathrm{P}<0.05$ levels. This indicates that the difference in score before and after administration of Play therapy.

Table 7:-mean standard deviation and comparison between pre and posttest on level of anxiety of parents among control group-

$\mathbf{N}=\mathbf{3 0}$

\begin{tabular}{|l|l|c|c|c|c|}
\hline S.no & Group & Pre anxiety score & Post anxiety score & Paired 't'test & \multirow{2}{*}{ P value } \\
\cline { 3 - 6 } & & Mean \pm SD & Mean \pm SD & & \\
\hline 1. & Control group & $32.40 \pm 12.4$ & $29.63 \pm 12.5$ & 2.592 & $.015^{*}$ \\
\hline
\end{tabular}

$\mathrm{Df}_{29}=\mathbf{2 . 0 4 5} *$ Significant

Table 7-, depicts that in control group mean pre test anxiety score of parents was 32.40 and mean post anxiety score was 29.63 respectively. From the mean scores it is clear that parents were in control group have lower anxiety score. The values are significant at $\mathrm{P}<0.05$ levels. This indicates that the difference in score before and after routine hospital treatment is significant in control group.

Table 7:- Mean Standard deviation and comparison scores of posttest anxiety level of parents.

$\mathbf{N}=60$

\begin{tabular}{|l|c|c|c|c|c|}
\hline \multirow{2}{*}{ S.NO } & GROUP & ANXIETY SCORE & t-calculated & TABLE & P \\
\cline { 3 - 3 } & & Malue & VALUE & VALUE \\
\hline 1. & Experimental group & $20.87 \pm 8.8$ & & & \\
\cline { 3 - 3 }. & Control group & $29.63 \pm 12.5$ & 3.115 & 2.00 & $\mathbf{0 . 0 0 3} *$ \\
\hline
\end{tabular}

$\mathbf{t}_{\mathbf{5 8}}=\mathbf{2 . 0 0} *$ Significant $\mathbf{p}<\mathbf{0 . 0 5}$

Table 7 shows that mean posttest anxiety score of parents in experimental group was 20.87 where as in control group mean post test anxiety score of parents was 29.63. This indicates that there was mean scores differences in experimental and control group. These values were significant at $\mathrm{P}<0.05$ levels.

Table 8:-Association between the pre test anxiety level score of hospitalized children's parents and selected demographic variables

$\mathrm{N}=60$

\begin{tabular}{|c|c|c|c|c|c|c|}
\hline \multirow[t]{3}{*}{ Selected variables } & \multicolumn{4}{|c|}{ Level of anxiety } & \multirow[t]{3}{*}{ Chi square } & \multirow[t]{3}{*}{ P value } \\
\hline & \multicolumn{2}{|c|}{ At or below the median } & \multicolumn{2}{|c|}{ Above the median } & & \\
\hline & No & $\%$ & No & $\%$ & & \\
\hline $\begin{array}{r}\text { Age of child } \\
3-5 \text { years } \\
6-8 \text { years }\end{array}$ & $\begin{array}{l}17 \\
15\end{array}$ & $\begin{array}{l}28 \\
25\end{array}$ & $\begin{array}{l}13 \\
15\end{array}$ & $\begin{array}{l}22 \\
25\end{array}$ & 0.268 & 0.60 \\
\hline $\begin{array}{l}\text { No of children in family } \\
\text { - } \quad 1-2 \\
\text { - } \quad 3 \text { and above }\end{array}$ & $\begin{array}{l}20 \\
12\end{array}$ & $\begin{array}{l}33 \\
20\end{array}$ & $\begin{array}{l}11 \\
17\end{array}$ & $\begin{array}{l}18 \\
29\end{array}$ & 3.19 & 0.07 \\
\hline $\begin{array}{c}\text { Education status of father } \\
\text { - } \quad \text { Graduate \& above } \\
\text { - } \quad \text { Illiterate-secondary }\end{array}$ & $\begin{array}{l}18 \\
14\end{array}$ & $\begin{array}{l}30 \\
23\end{array}$ & $\begin{array}{l}07 \\
21\end{array}$ & $\begin{array}{l}12 \\
35\end{array}$ & 5.933 & 0.01 \\
\hline
\end{tabular}




\begin{tabular}{|c|c|c|c|c|c|c|}
\hline $\begin{array}{c}\text { Education status of mother } \\
\bullet \quad \text { Graduate } \\
\bullet \quad \text { Illiterate-secondary } \\
\end{array}$ & $\begin{array}{l}16 \\
16 \\
\end{array}$ & $\begin{array}{l}27 \\
27 \\
\end{array}$ & $\begin{array}{l}07 \\
21 \\
\end{array}$ & $\begin{array}{l}12 \\
35 \\
\end{array}$ & 3.94 & 0.04 \\
\hline $\begin{array}{c}\text { Occupation of father } \\
\text { - Professional } \\
\text { - } \quad \text { Nonprofessional }\end{array}$ & $\begin{array}{l}19 \\
13\end{array}$ & $\begin{array}{l}32 \\
22\end{array}$ & $\begin{array}{l}08 \\
20\end{array}$ & $\begin{array}{l}13 \\
33\end{array}$ & 5.725 & 0.016 \\
\hline $\begin{array}{cl}\text { Occupation of mother } \\
\text { - } & \text { Professional } \\
\text { - } & \text { Nonprofessional } \\
\end{array}$ & $\begin{array}{l}10 \\
22 \\
\end{array}$ & $\begin{array}{l}17 \\
37 \\
\end{array}$ & $\begin{array}{l}02 \\
26 \\
\end{array}$ & $\begin{array}{l}03 \\
43 \\
\end{array}$ & 5.424 & 0.02 \\
\hline $\begin{array}{l}\text { Previous experience of } \\
\text { hospital } \\
\text { - Never- Once } \\
\text { - Twice and above }\end{array}$ & $\begin{array}{l}23 \\
09\end{array}$ & $\begin{array}{l}38 \\
15\end{array}$ & $\begin{array}{l}12 \\
16\end{array}$ & $\begin{array}{l}20 \\
27\end{array}$ & 5.176 & 0.02 \\
\hline
\end{tabular}

df $_{1}=3.84 P<0.05$

Table-8 depict that, the variable age of child, gender of child, number of children, with computed chi-square respectively shows no association with pretest anxiety level score accept the occupation of father, occupation of mother, education status of father, education of mother and previous experience of hospital have association with pretest anxiety level scores at 0.05 level of significance.

\section{Nursing Implication:-}

The nursing implications are discussed under nursing practice, nursing education, nursing administration and nursing research

The finding of the study clearly highlights the importance of the play therapy to the hospitalized children. The nursing personnel both in hospital and community can conduct educational programme and Motivate to the nursing student to apply play therapy when they are in clinical practice. Conduct in-service education regarding play therapy in pediatric nursing setting. This present study conducted by the investigator can be a source of review of literature for others, who are intending to conduct study on effectiveness of play therapy on physiological parameters of children and in reducing the anxiety of hospitalized children's parents

\section{Conclusion:-}

From the findings of the study, it can be concluded that the administered of play therapy was effective as a method, to improve the physiological parameters of the children between 3-8 years and reduce the level of anxiety of their parents .. The finding shows that the mean post test anxiety score is less than the mean pretest anxiety score of parents. And there is improvement in physiological parameters of children. So play therapy is effective for hospitalized children.

\section{References:-}

i. Kemper KJ., Danhhauer SC. Music as therapy. South Med J. March 2005; 98(3

ii. Harrison Wein. Stress and Disease: New Perspectives. Scientific American journal. 2003 September. 360;15-21

iii. Glavas M, Weinberg J. "Play Science - the Patterns of Play". Journal of National Institute for Play. 2009;11-21.

iv. Rimple sharma, Essentials of Pediatric Nursing for B Sc \& Post Basic B Sc Nursing students, 1st Edition 2013, Jaypee Brothers Medical publisher (P) Ltd. Pg. No. 125-127.

v. Brunner L, Sudarth S. Text book of Medical Nursing. Philadelphia. Lippincott Company. $\quad$ 2000; 62.

${ }^{\text {vi }}$. Harrison Wein. Stress and Disease: New Perspectives. Scientific American journal. 2003 September. 360;15-21 